\title{
EXTREME POINTS AND CONVOLUTION PROPERTIES OF SOME CLASSES OF MULTIVALENT FUNCTIONS
}

\author{
O. P. AHUJA \\ Division of Mathematics \\ National Institute of Education \\ Nanyang Technological University \\ 469 Bukit Timah Road \\ Singapore 259756, REPUBLIC OF SINGAPORE
}

(Received April 5, 1988 and in revised form June 26, 1991)

\begin{abstract}
This paper deals with the extreme points of closed convex hulls of the classes of multivalent functions related to Ruscheweyh derivatives and then these are used to determine the coefficient bounds Finally, we investigate convolution conditions and other properties of the functions in these classes
\end{abstract}

KEY WORDS AND PHRASES: Multivalent, Hadamard product, starlike, Ruscheweyh derivative 1990 AMS SUBJECT CLASSIFICATION CODES: Primary 30C45, 30C99, Secondary 30C55

\section{INTRODUCTION}

An analytic $s$-function is said to be $p$-valent if it assumes each value not more than $p$-times and some value exactly $p$-times Let $M(p), p \geq 1$ integer, denote the family of all functions of the form

$$
f(z)=z^{p}+\sum_{k=p+1}^{\infty} a_{k} z^{k}
$$

which are analytic and $p$-valent in the unit disk $\triangle=\{z:|z|<1\} \quad$ Let $S_{p}^{*}(\alpha)$ denote the class of functions of the form (ll 11$)$ which satisfy the conditions

$$
\operatorname{Re}\left(\frac{z f^{\prime}(z)}{f(z)}\right)>p \alpha, \quad \text { and } \quad \int_{0}^{2 \pi} \operatorname{Re}\left(\frac{z f^{\prime}(z)}{f(z)}\right) d \theta=2 \pi p
$$

for $0 \leq \alpha<1$ and $z \in \triangle$ A function in $S_{p}^{*}(\alpha)$ is called a $p$-valent starlike of order $\alpha$ in $\triangle$ The class $S_{p}^{*}\left(\alpha^{\prime}\right)$, where $\alpha^{\prime}=\alpha p, 0 \leq \alpha^{\prime}<p$, was introduced by Goluzina [1] A function $f$ of the form (11) is said to be in $C_{p}(\alpha)$ if $z f^{\prime}(z) / p$ is in $S_{p}^{*}(\alpha)$ A function in $C_{p}(\alpha)$ is called a $p$-valent convex of order $\alpha$ in $\triangle$ We observe that $S_{p}^{*}(\alpha) \subset S_{p}^{*}(0)=S_{p}^{*}, C_{p}(\alpha) \subset C_{p}(0)=C_{p} \quad$ Goodman [2] introduced the classes $S_{p}^{*}$ and $C_{p}$ In the same paper, he observed that these are subclasses of $M(p)$ Besides, note that $S_{1}^{*}(\alpha)=S^{*}(\alpha)$ and $C_{1}(\alpha)=C(\alpha)$, where $S^{*}(\alpha)$ and $C(\alpha)$ consist of the functions which are, respectively, starlike of order $\alpha$ and convex of order $\alpha$ in $\triangle$, see, for example [3] Also, we notice that there are different ways of extending starlike and convex concepts to $p$-valent functions and Hummel [4] has made an extensive study of the various possibilities

If $f(z)=z^{p}+\sum_{k=p+1}^{\infty} a_{k} z^{k}$ and $g(z)=z^{p}+\sum_{k=p+1}^{\infty} b_{k} z^{k}$ are two power series in $\triangle$, then its Hadamard product is defined as $(f * g)(z)=z^{p}+\sum_{k=p+1}^{\infty} \begin{gathered}k=p+1 \\ a_{k} b_{k} z^{k} \text { in }\end{gathered}$ For $f \in M(p)$, we write 


$$
D^{\prime \prime} p^{\prime} f(z)=\frac{z^{p}}{(1-z)^{n} p} * f(z), \quad n \geq-p+1
$$

The operator $D^{n \cdot p} 1 f$ is called the $(n+p-1)$ th order Ruscheweyh derivative of $f$ Let $R_{n}(p, \alpha)$ denote the subclasses of functions $f$ in $M(p)$ which satisfy the conditions

$$
\operatorname{Re}\left(\frac{z\left(D^{n \cdot p}{ }^{1} f(z)\right)^{\prime}}{D^{n}{ }^{1}{ }^{1} f(z)}\right)>p \alpha \quad \text { and } \quad \int_{0}^{2 i} \operatorname{Re}\left(\frac{z\left(D^{n+p}{ }^{1} f(z)\right)^{\prime}}{D^{n+p}{ }^{1} f(z)}\right) d \theta=2 \pi p
$$

for $0 \leq \alpha<1$ and $z \in \triangle$ The condition (14b) implies that $D^{n+p-1} f(z)$ has $p$ roots in $\triangle$ We observe that $R_{p}{ }_{1}(p, \alpha)=S_{p}^{*}(\alpha), R_{0}(1, \alpha)=S^{*}(\alpha)$, and $R_{1}(1, \alpha)=C(\alpha)$ The class $R_{n}(1, \alpha)=R_{n}(\alpha)$ was introduced and studied by the author [5] Moreover, $R_{n}(p, \alpha) \subset R_{n}(p, 0) \subset K_{n}(p)$ for $n \geq p$, where

$$
K_{n}(p)=\left\{f \in M(p): \operatorname{Re} \frac{D^{n ; p} f(z)}{D^{n+p-1} f(z)}>1 / 2, z \in \triangle\right\}
$$

are subclasses of $M(p)$ studied by Goel and Sohi [6] Note that $K_{n}(1)$ was introduced by Ruscheweyh [7]

The fundamental viewpoint of considering convex hulls and extreme points of $S^{*}(\alpha)$ and $C(\alpha)$ were first given in [8] and [9] The author and Silverman [10] have studied the extreme points of the closed convex hull of $R_{n}(\alpha)$ Earlier, some of the concepts of convex hulls and extreme points were extended to - multivalent functions in [11-13], and others

In the present paper, we determine the extreme points of the closed convex hulls of $R_{n}(p, \alpha)$ and $K_{n}(p)$ These are then used to determine the coefficient bounds Finally, we investigate convolution conditions and other properties of the functions in $R_{n}(p, \alpha)$

In the sequel, we denote the closed convex hull of a family $F$ by $\operatorname{co} F$ Also, let $E(\operatorname{co} F)$ denote the set of all extreme points of $F$

\section{EXTREME POINTS}

LEMMA 2.1. [12] $E\left(\cos S_{p}^{*}(\alpha)\right)$ consists of the functions given by

$$
\frac{z^{p}}{(1-x z)^{2(1-\alpha) p}}=z^{p}+\sum_{k=p+1}^{\infty} \frac{(2 p-2 \alpha p)_{k-p} x^{k-p}}{(k-p) !} z^{k}
$$

$|x|=1, z \in \triangle$, where $(a)_{k}=a(a+1)(a+2) \ldots(a+k-1)$

THEOREM 2.1. The extreme points of $\operatorname{co} R_{n}(p, \alpha), 0 \leq \alpha<1$, are given by the functions

$$
f_{x}(z)=z^{p}+\sum_{k=p+1}^{\infty} \frac{(2 p-2 \alpha p)_{k-p}(n+p-1) !}{(k+n-1) !} x^{k-p} z^{k}, \quad|x|=1, \quad z \in \triangle .
$$

PROOF. We first notice that the operator $D^{n+p-1}: f \rightarrow D^{n+p-1} f$ is an isomorphism from $R_{n}(p, \alpha)$ to $S_{p}^{*}(\alpha)$ and consequently it preserves extreme points Also, we observe that

$$
D^{n+p-1} f(z)=\frac{z^{p}}{(1-z)^{n+p}} * f(z)=z^{p}+\sum_{k=p+1}^{\infty}\left(\begin{array}{c}
n+k-1 \\
n+p-1
\end{array}\right) a_{k} z^{k} .
$$

Therefore, from Lemma 21 we find that the extreme points of $R_{n}(p, \alpha)$ are given by

$$
z^{p}+\sum_{k=p+1}^{\infty}\left(\begin{array}{c}
n+k-1 \\
n+p-1
\end{array}\right)^{-1} \frac{(2 p-2 \alpha p)_{k-p}}{(k-p) !} x^{k-p} z^{k} .
$$

This simplifies to (2 2) and the proof is complete

REMARK 2.1. The special case of $p=1$ in Theorem 21 yields the extreme points of $c o R_{n}(\alpha)$ found in [10] 
REMARK 2.2. Lettıng $n=-p+1$ and $a=0$ in Theorem 21 , we obtain the extreme points of co $S_{p}$ found by Hallenbeck and Livingston [11]

COROLLARY 2.1. If $f(z)=z^{p}+\sum_{k}^{x} a_{k} z^{h}$ is in $R_{n}(p, \alpha)$, then

$$
\left|a_{h}\right| \leq \frac{(2 p-2 \alpha p)_{h}(n+p-1) !}{(k+n-1)^{\prime}}, \quad k \geq p+1
$$

with equality for

$$
f_{\perp}(z)=z^{p}+\sum_{k=1}^{x} \frac{(2 p-2 \alpha p)_{h}(n+p-1) !}{(k+n-1)^{\prime}} x^{k} z^{p} z^{k}, \quad|x|=1, \quad z \in \Delta .
$$

COROLLARY 2.2. If $f(z)=z^{p}+\sum_{h=p+1}^{x} a_{k} z^{h}$ is in $S_{p}^{*}$, then

$$
\left|a_{k}\right| \leq \frac{(2 p)(2 p+1) \ldots(p+k-1)}{(k-p) !}, \quad k \geq p+1 .
$$

The above result of Goodman [2] may be found by letting $\alpha=0, n=-p+1$ in Corollary 21

COROLLARY 2.3. If $f(z)=z^{p}+\sum_{k=p+1}^{\infty} a_{k} z^{k}$ is in $R_{n}(p, \alpha)$, then

$$
|f(z)| \leq r^{p}+\sum_{k=p+1}^{\infty} \frac{(2 p-2 \alpha p)_{k-p}(n+p-1) !}{(k+n-1) !} r^{k},
$$

with equality for $f_{x}(z)$ at $z=\bar{x} r$.

In [10], the author and Silverman found the extreme points of the closed convex hull of $K_{n}(p)$, when $p=1$ In the next theorem, we find the corresponding result when $p \geq 1$

LEMMA 2.2. [6] $K_{n}(p) \subset K_{n-1}(p)$ for every $n \geq-p+1$

THEOREM 2.2. The extreme points of $c o K_{n}(p)$ are

$$
\left\{\frac{z^{p}}{1-x z}=z^{p}+\sum_{k=p+1}^{\infty} x^{k-p} z^{k}:|x|=1, z \in \triangle\right\} \text { for all } n \geq-p+1 .
$$

PROOF. For $g(z)=z^{p} /(1-x z)$, we observe that

$$
D^{n+p-1} g(z)=\frac{z^{p}}{(1-x z)^{n+p}} * \frac{z^{p}}{1-x z}=\frac{z^{p}}{(1-x z)^{n+p}} .
$$

Therefore,

$$
\operatorname{Re} \frac{D^{n+p} g(z)}{D^{n+p-1} g(z)}=\operatorname{Re} \frac{1}{1-x z}>1 / 2
$$

It implies that $g \in K_{n}(p)$ for every $n \geq-p+1$ We thus have

$$
\left\{\frac{z^{p}}{1-x z}:|x|=1, z \in \Delta\right\} \subset K_{n}(p) .
$$

But by Lemma $22, K_{n}(p) \subset K_{-p+1}(p)$ Since

$$
\begin{aligned}
K_{-p+1}(p) & =\left\{f \in M(p): \operatorname{Re} \frac{D^{1} f}{D^{2} f}>\frac{1}{2}\right\} \\
& =\left\{f \in M(p): \operatorname{Re} \frac{z f^{\prime}-(p-1) f}{f}>1 / 2\right\}=S_{p}^{*}\left(\frac{2 p-1}{2 p}\right),
\end{aligned}
$$

we have 


$$
\left\{\frac{z^{p}}{1-x z} \cdot|x|=1, z \in \triangle\right\} \subset S_{p}^{*}\left(\frac{2 p-1}{2 p}\right)
$$

On the other hand, the extreme points of $\cos S_{p}^{*}\left(\frac{2 p 1}{2 p}\right)$ are $\left\{\frac{z^{i}}{1 z} \cdot|x|=1, z \in \triangle\right\}$, from Lemma 21 , and the result follows

COROLLARY 2.4 If $f(z)=z^{p}+\sum_{k} \sum_{p, 1}^{x} a_{h} z^{k} \in K_{n}(p)$, then $\left|a_{k}\right| \leq 1$ for $k \geq p+1$

\section{INCLUSION RELATIONS}

LEMMA 3.1. [14] Let $\omega$ be a non-constant and analytic function in $|z|\langle r<1, \omega(0)=0$ If $|\omega|$ attains its maximum value on the circle $|z|=r$ at $z_{0}$, then $z_{0} \omega^{\prime}\left(z_{0}\right)=k \omega\left(z_{0}\right)$, where $k(\geq 1)$ is any real number

THEOREM 3.1. $R_{n \cdot 1}(p, \alpha) \subset R_{n}(p, \alpha)$ for all $\alpha(0 \leq \alpha<1)$, and $n \geq-p+1$

PROOF. Let $f \in R_{n, p}(p, \alpha)$ Define an analytic function $\omega(z)$ in $\triangle$ such that $\omega(0)=0$, $\omega(z) \neq-1$ for all $z \in \triangle$ by

$$
\frac{z\left(D^{n \cdot p}{ }^{\prime} f(z)\right)^{\prime}}{D^{n \cdot p \cdot} \cdot 1(z)}=p\left(\frac{1+(2 \alpha-1) \omega(z)}{1+\omega(z)}\right) .
$$

Using the identity

$$
z\left(D^{n+p-1} f(z)\right)^{\prime}=(n+p) D^{n+p} f(z)-n D^{n+p-1} f(z),
$$

we can rewrite $\left(\begin{array}{ll}3 & 1\end{array}\right)$ as

$$
\frac{D^{n+p} f(z)}{D^{n+p-1} f(z)}=\frac{n+p+((2 \alpha-1) p+n) \omega(z)}{(n+p)(1+\omega(z))}
$$

Taking logarithmic differentiation of (3 3), we get

$$
\frac{z\left(D^{n+p} f(z)\right)^{\prime}}{D^{n+p} f(z)}=p\left(\frac{1+(2 \alpha-1) \omega(z)}{1+\omega(z)}\right)-\frac{2 p(1-\alpha) z \omega^{\prime}(z)}{(1+\omega(z))(n+p+(n+(2 \alpha-1) p) \omega(z))} .
$$

We claim that $|w(z)|<1$ for all $z \in \triangle$ For otherwise, by Lemma 31 , there exists a point $z_{0} \in \triangle$ such that $z_{0} \omega^{\prime}(z)=k \omega\left(z_{0}\right)$ with $\left|\omega\left(z_{0}\right)\right|=1$ and $k \geq 1$ Applying this result to (34), we obtain

$$
\operatorname{Re}\left(\frac{z_{0}\left(D^{n+p} f\left(z_{0}\right)\right)^{\prime}}{D^{n+p} f\left(z_{0}\right)}\right) \leq p \alpha-\frac{k p(1-\alpha)}{2(n+\alpha p)} \leq p \alpha \text { for each } n \geq-p+1
$$

This proves that $f \notin R_{n+1}(p, \alpha)$, which contradicts the hypothesis We thus conclude that $|\omega(z)|<1$ for all $z \in \triangle$ and hence $f \in R_{n}(p, \alpha)$ This completes the proof

In view of Theorem 31 , it immediately follows that $R_{n}(p, \alpha) \subset S_{p}^{*}(\alpha)$ for all $n \geq-p+1$ For $\alpha$ fixed and $n=n(\alpha)$ sufficiently large, we shall now show that $R_{n}(p, 0) \subset C_{p}(\alpha)$ We need the following

LEMMA 3.2. Let $f(z)=z^{p}+\sum_{k=p+1}^{\infty} a_{k} z^{k} \in M(p)$, and let $0 \leq \alpha<1$ The function $f$ is in $C_{p}(\alpha)$ if

$$
\sum_{k=p+1}^{\infty} k(k-\alpha p)\left|a_{k}\right| \leq p^{2}(1-\alpha) .
$$

PROOF. It suffices to show that

$$
\left|1+\frac{z f^{\prime \prime}(z)}{f^{\prime}(z)}-p\right| \leq p(1-\alpha) .
$$

We have 


$$
\begin{aligned}
\left|z f^{\prime \prime}(z)-(p-1) f^{\prime}(z)\right|-p(1-a)\left|f^{\prime}(z)\right| \\
\quad \leq\left|\sum_{h p 1}^{x} k(k-p) a_{h}\right|-p(1-a)\left(p-\sum_{h}^{x} k\left|a_{h}\right|\right) \\
\quad \leq \sum_{h=1}^{x} k(k-p)\left|a_{h}\right|-p^{2}(1-a)+p(1-\alpha) \sum_{h \cdot 1}^{x} k\left|a_{h}\right| \\
\quad=\sum_{k}^{x} k(k-\alpha p)\left|a_{h}\right|-p^{2}(1-\alpha) \leq 0,
\end{aligned}
$$

which proves (3 6) This completes the proof

THEOREM 3.2. For fixed $\alpha, 0 \leq \alpha<1, p \geq 1$ integer

$$
R_{n}(p, 0) \subset C_{p}(\alpha) \text { for any } n \geq n_{0}=\frac{2(p+1)^{4}}{p(1-\alpha)} .
$$

PROOF. Let $f(z)=z^{p}+\sum_{k=p+1}^{\infty} a_{k} z^{k}$ be in $R_{n}(p, 0)$ We observe that

$$
f \in R_{n}(p, 0) \Longleftrightarrow D^{n+p-1} f \in S_{p}^{*} .
$$

In view of (23), and as a consequence of Corollary 22 of Theorem 21 , we obtain

$$
\left|\left(\begin{array}{c}
n+k-1 \\
n+p-1
\end{array}\right) a_{k}\right| \leq \frac{(2 p)_{k-p}}{(k-p) !}
$$

which yields

$$
\left|a_{k}\right| \leq \frac{(2 p)_{k-p}}{(k-p) !}\left(\begin{array}{c}
n+k-1 \\
n+p-1
\end{array}\right)^{-1} \quad \text { for all } \quad k \geq p+1 .
$$

Therefore, because of Lemma 32 , we only need to prove that

$$
\sum_{k=p+1}^{\infty} k^{2}\left|a_{k}\right| \leq \sum_{k=p+1}^{\infty} k^{2} \frac{(2 p)_{k-p}}{(k-p) !}\left(\begin{array}{c}
n+k-1 \\
n+p-1
\end{array}\right)^{-1} \leq p^{2}(1-\alpha) \text { for all } n \geq n_{0} .
$$

Since $\sum_{k=p+1}^{\infty}\left(1 / k^{2}\right)<1$, it suffices to establish that

$$
\sum_{k=p+1}^{\infty} \frac{k^{2}(2 p)_{k-p}}{(k-p) !}\left(\begin{array}{c}
n+k-1 \\
n+p-1
\end{array}\right)^{-1} \leq p^{2}(1-\alpha) \sum_{k=p+1}^{\infty}\left(1 / k^{2}\right)
$$

for all $n \geq n_{0} \quad$ We notice that (3 9) holds if

$$
d_{k}=\frac{k^{4}(2 p)_{k-p}}{(k-p) !}\left(\begin{array}{c}
n+k-1 \\
n+p-1
\end{array}\right)^{-1} \leq p^{2}(1-\alpha)
$$

for all $n \geq n_{0}, k \geq p+1$ But

$$
\left(\begin{array}{c}
n+k-1 \\
n+p-1
\end{array}\right)^{-1}=\frac{(n+p-1) !(k-p) !}{(n+k-1) !}
$$

is a decreasing function of $n(\geq-p+1)$ Therefore, we need only prove (3 10) for $n=n_{0}$ Since for $n=n_{0}$,

$$
d_{p+1}=\frac{2 p(p+1)^{4}}{n_{0}+p} \leq p^{2}(1-\alpha)
$$


is true for all $p \geq 1$, it follows that $\left(\begin{array}{l}3 \\ 10\end{array}\right)$ holds for $k=p+1$ Thus the proof of the theorem will be completed by proving that $d_{h}$ is a decreasing function of $k(\geq p+1)$ for $n=n_{(1)}$, that is, if

$$
\frac{d_{h} 1}{d_{h}}=\frac{(k+1)^{\prime}(k+p)}{k^{\prime}\left(k+n_{0}\right)} \leq 1 \text {, }
$$

which is equivalent to

$$
g(k)=k^{\prime}\left(n_{0}-4-p\right)-k^{3}(6+4 p)-k^{2}(4+6 p)-k(1+4 p)-p \geq 0
$$

Since

$$
n_{0}-4-p \geq \frac{2(p+1)^{4}-p(4+p)}{p}
$$

we have

$$
g(k) \geq\left(\frac{2(p+1)^{4}}{p}-15-16 p\right) k^{4}=\left[\frac{2(p+1)}{p}\left((p+1)^{3}-8 p\right)+1\right] k^{4}>0
$$

for all $p \geq 1$ The proof is complete

\section{CONVOLUTION CONDITIONS}

LEMMA 4.1. Let $f \in M(p)$ Then $f \in S_{p}^{*}(\alpha), 0 \leq \alpha<1, p \geq 1$, if and only if $f(z) *$ $\left[\left(z^{p}+B z^{p+1}\right) /(1-z)^{2}\right] \neq 0(0 \leq|z|<1,|x|=1)$, where

$$
B=\frac{x+1-2 p(1-\alpha)}{2 p(1-\alpha)}
$$

PROOF. Since $z f^{\prime}(z) / f(z)$ at $z=0$ is $p$, therefore

$$
\operatorname{Re}\left(\frac{\frac{z f^{\prime}(z)}{f(z)}-\alpha p}{p-\alpha p}\right)>0
$$

which is equivalent to

$$
\frac{\frac{z f^{\prime}(z)}{f(z)}-\alpha p}{p-\alpha p} \neq \frac{x-1}{x+1}, \quad|x|=1, \quad x \neq-1
$$

This simplifies to

$$
\left(z f^{\prime}(z)-\alpha p f(z)\right)(x+1)-(p-\alpha p)(x-1) f(z) \neq 0
$$

in the annulus $\rho<|z|<1$ for some $\rho(0<\rho<1)$ For $f(z)=z^{p}+\sum_{k=p+1}^{\infty} a_{k} z^{k}$, it is easy to show that

$$
f(z) * \frac{z^{p}}{(1-z)^{2}}=z^{p}+\sum_{k=p+1}^{\infty}(k-p+1) a_{k} z^{k}=z f^{\prime}(z)-(p-1) f(z),
$$

and

$$
f(z) * \frac{z^{p}}{1-z}=f(z)
$$

Therefore, $(42)$ is equivalent to

$$
f(z) *\left[\left\{\frac{z^{p}}{(1-z)^{2}}+(p-1) \frac{z^{p}}{1-z}-\alpha p \frac{z^{p}}{1-z}\right\}(x+1)-(p-\alpha p)(x-1) \frac{z^{p}}{1-z}\right] \neq 0
$$

that is 


$$
f(z) *\left(\frac{2 p(1-a) z^{p}+(x+1-2 p(1-a)) z^{p} \quad 1}{(1-z)^{2}}\right) \neq 0
$$

This proves $\left(\begin{array}{ll}4 & 1\end{array}\right)$

THEOREM 4.1. The function $f$ is in $R_{t}(p, a)$ if and only if

$$
f(z) *\left(\frac{z^{p}+\frac{(n p) i n p(2 n 1)}{2 p(n)} z^{p} 1}{(1-z)^{n-1}}\right) \neq 0
$$

for $0<|z|<1,|x|=1$.

PROOF. Since $f \in R_{n}(p, a)$ if and only if $D^{n \cdot p}$ ' $f \in S_{p}^{*}(a)$, an application of (1 3$)$ to Lemma 41 yields

$$
f(z) *\left(h(z) *\left(\frac{z^{p}}{(1-z)^{2}}+\frac{B z^{p \cdot 1}}{(1-z)^{2}}\right)\right) \neq 0
$$

where $h(z)=z^{p} /(1-z)^{n+p}$ But in view of (4 3) and (4 4), we may write

$$
\begin{aligned}
h(z) *\left(\frac{z^{p}}{(1-z)^{2}}+\frac{B z^{p 1}}{(1-z)^{2}}\right) & =h(z) * \frac{z^{p}}{(1-z)^{2}}+B h(z) * \frac{z^{p+1}}{(1-z)^{2}} \\
& =z h^{\prime}(z)-(p-1) h(z)+B\left(z h^{\prime}(z)-p h(z)\right) \\
& =(B+1) z h^{\prime}(z)-(p-1+B p) h(z) \\
& =(B+1)\left(\frac{p z^{p}}{(1-z)^{n+p}}+\frac{(n+p) z^{p+1}}{(1-z)^{n+p+1}}\right)-(p-1+B p) \frac{z^{p}}{(1-z)^{n \cdot p}} \\
& =\frac{z^{p}+(-1+(B+1)(n+p)) z^{p+1}}{(1-z)^{n+p+1}} .
\end{aligned}
$$

Substituting the value of $B$ from (4 1), simplifying, the result then follows from (4 6)

\section{CONCLUDING COMMENTS}

It would be possible to obtain additional information and solutions to extremal problems for the family $R_{n}(p, \alpha)$ if one gets $f_{x}(z)$ in $(22)$ into closed form For example, using the closed form, viz $z^{p} /(1-x z)^{2 p}$, for $n=-p+1$ and $\alpha=0$, Hallenbeck and Livingston [11] found coefficient estimates for functions majorized by or subordinate to functions in $S_{p}^{*}$ Note that the closed form of (2 2) even for the special case of $p=1$ is an open problem (see, [10])

\section{REFERENCES}

[1] GOLUZINA, E G, On the coefficients of a class of functions regular in a disk and having an integral representation in it, J. Soviet Math. 2 (6) (1976), 606-617

[2] GOODMAN, A W, On the Schwarz-Christoffel transformation and p-valent functions, Trans. Amer. Math. Soc. 68 (1950), 204-223

[3] GOODMAN, A W , Univalent Functions, Mariner Publishing Company, Inc , 1983

[4] HUMMEL, J A , Multivalent starlike functions, J. Analyse Math. 18 (1967), 133-160

[5] AHUJA, O P, Integral operators of certain univalent functions, Internat. J. Math. \& Math. Scl. 8 (4) (1985), 653-662

[6] GOEL, R M and SOHI, N S, A new criterion for p-valent functions, Proc. Amer. Math. Soc. 78 (3) (1980), 353-357

[7] RUSCHEWEYH, S, New criteria for univalent functions, Proc. Amer. Math. Soc. 49 (1975), 109115

[8] BRICKMAN, L, MacGREGOR, T H and WILKEN, D R, Convex hulls of some classical families of univalent functions, Trans. Amer. Math. Soc 156 (1971), 91-107 
[9] BRICKMAN, L, HALLENBECK, D J, MacGREGOR, T H and WILKEN, D R, Convex hulls and extreme points of families of starlike and convex mappings, Irans. Amer. Math. Soc. 185 (1973), 413-428

[10] AHUJA, O P and SILVERMAN, H, Function classes related to Ruscheweyh derivatives, J. Aust. Math. Soc. 47 (1989), 438-444

[11] HALLENBECK, D J and LIVINGSTON, A E , Applications of extreme point theory to classes of multivalent functions, Trans. Amer. Math. Soc. 221 (2) (1976), 339-359

[12] KAPOOR, GP and MISHRA, A K, Convex hulls and extreme points of some classes of multivalent functions, J. Math. Anal. Appl. 87 (1982), 116-126

[13] MacGREGOR, T H, Applications of extreme point theory to univalent functions, Mich. Math. J. 19 (1972), 361-376

[14] JACK, I S, Functions starlike and convex of order $\alpha$, J. London Math. Soc. 3 (1971), 469-474 


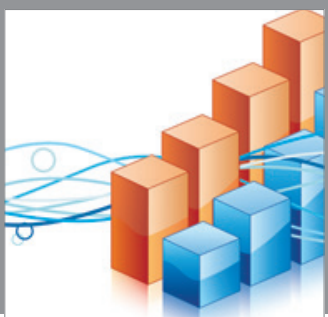

Advances in

Operations Research

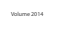

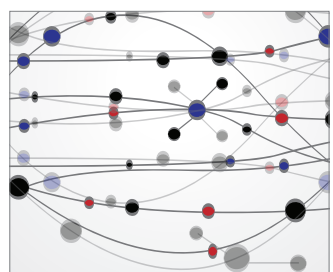

\section{The Scientific} World Journal
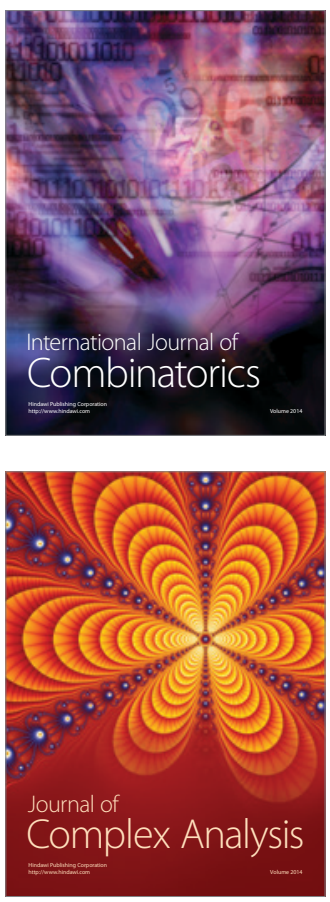

International Journal of

Mathematics and

Mathematical

Sciences
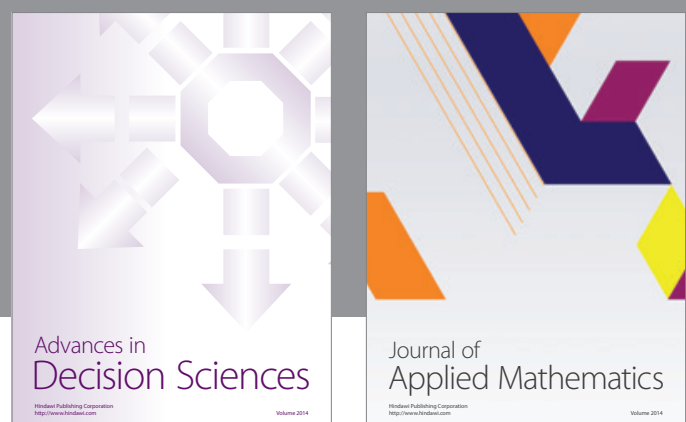

Journal of

Applied Mathematics
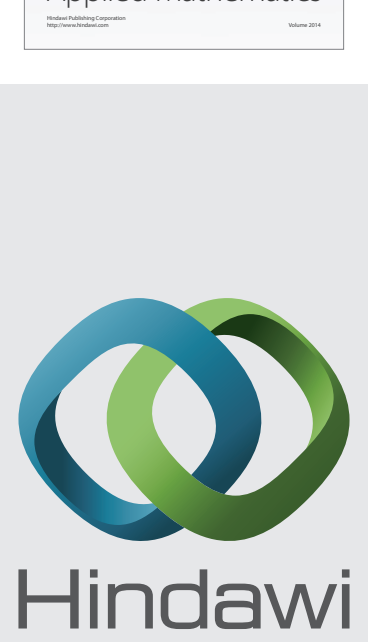

Submit your manuscripts at http://www.hindawi.com
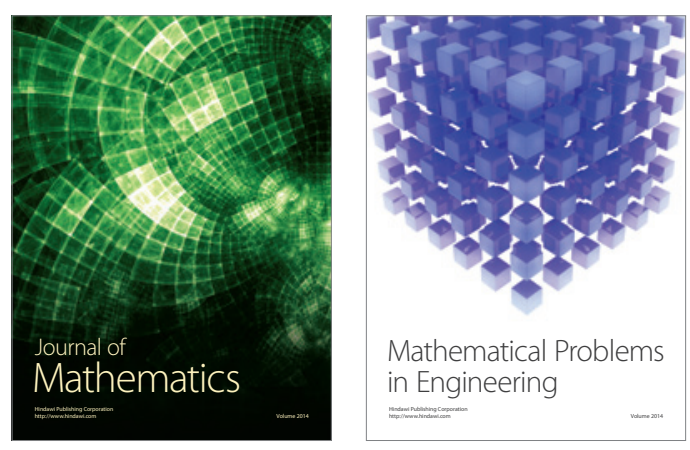

Mathematical Problems in Engineering
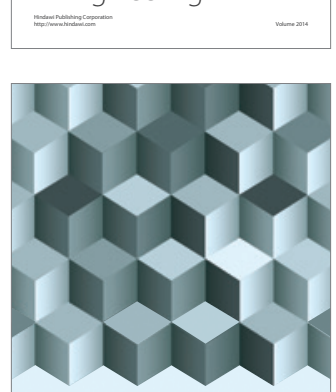

Journal of

Function Spaces
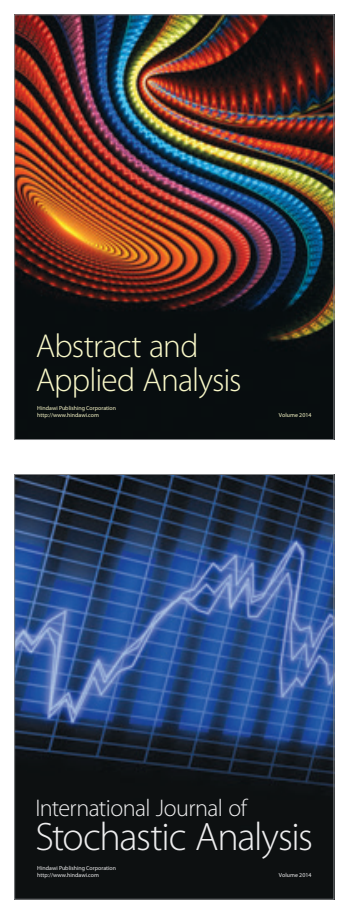

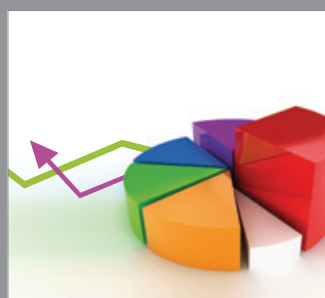

ournal of

Probability and Statistics

Promensencen
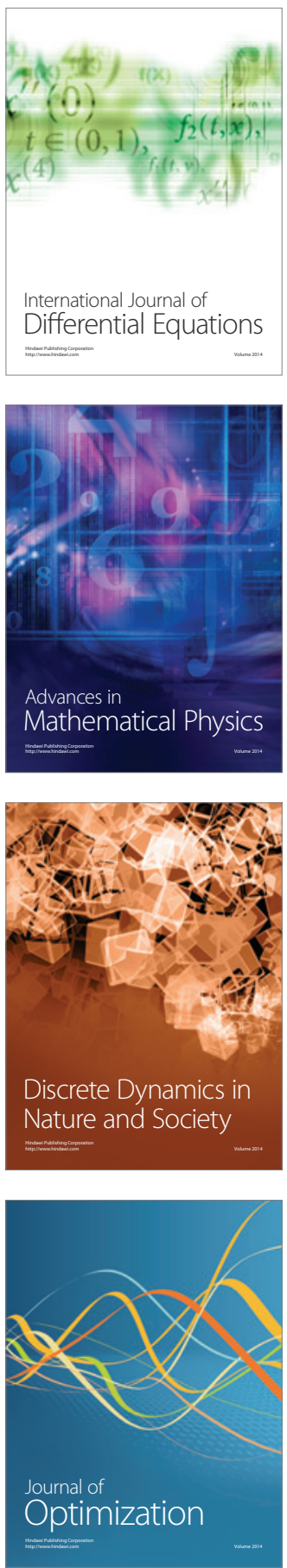